\title{
Sistema de neutralización dinámica en la columna lumbar. SISTEMA DYNESYS Experiencia en 94 casos
}

\author{
M. Bordes-Monmeneu; V. Bordes-García; F. Rodrigo-Baeza y D. Sáez
}

Hospital 9 de Octubre. Valencia.

Resumen

Objetivo. Valorar los resultados obtenidos con la utilización del sistema Dynesys (Dynamic Neutralización System for the spine), en un grupo de 94 pacientes, como nuevo concepto para el tratamiento de la patología degenerativa lumbar basado en la estabilización lumbar con conservación de la función articular en contra de la restricción de la artrodesis clásica.

Material y métodos. Presentamos 94 pacientes en los que se utilizó este sistema de los cuales 62 fueron hombres y 32 mujeres con una edad media de 46.4 años. La patología de los pacientes fue de hernia discal en 27 casos, discopatía degenerativa en 54 casos y estenosis de canal en 13 casos. Se realizó un seguimiento entre 14 t 24 meses valorando la clínica según la escala de Oswestry y la reincorporación al trabajo.

Resultados. Los resultados en la escala de Oswestry fue del $\mathbf{2 1 . 4 \%}$ definitivo con respecto al $\mathbf{5 6 . 8 \%}$ previo al tratamiento y la reincorporación al trabajo fue del $82 \%$. El cuadro ciático ha remitido prácticamente en todos los casos, así como la lumbalgia y una mejoría del $60 \%$ en casos de claudicación. Como complicaciones reseñamos dos casos debidas a la técnica, con malposición de tornillos en un caso y rotura pedicular en otro, dos casos de seroma subcutáneo y dos infecciones subclínicas tardías.

Conclusiones. La neutralización dinámica obtenida mediante este sistema, no debe ser considerada como una artrodesis. El tratamiento con Dynesys amplía la población de pacientes en los que se puede intervenir a aquellos que en principio no son tributarios de una fijación estándar, pero que planteaban dudas al cirujano al aplicar técnicas sin apoyo instrumental, incorporando el concepto de funcionalidad frente a la restricción del movimiento. Se puede definir como una prótesis díscal colocada extra-discalmente. Hemos obtenido un buen resultado en la mayoría de nuestros pacientes, aunque pensamos que es preciso ampliar el tiempo de seguimiento.

Recibido: 24-09-04. Aceptado: 27-01-05
PALABRAS CLAVE: Inestabilidad lumbar. Patología degenerativa lumbo-sacra. Estabilización dinámica. Sistema de neutralización. Dynesys.

Summary

Objective. To assess the results obtained using the Dynesys system (Dynamic Neutralisation System for the spine), in a group of 94 patients This new system for treating lumbar degenerative pathologie is based on lumbar stabilisation and preservation of articular function, as opposed to traditional arthrodesis restrictions.

Material and methods. We analyze series of 94 patiens in whom this system was used. 62 were males and 32 females with an average age of 46,4 years. The pathologies treated were disc herniation in 27 cases, degenerative discopathy in 54 cases and lumbar channel stenosis in 13 cases. Follow-up was carried out between 14 and 24 months, assessing the clinical picture according to the Oswestry scale and the return to work.

Results. The final results on the Oswestry scale were $21,4 \%$ with respect to $56,8 \%$ prior to the treatment and the return to work was $82 \%$. There was a remission of the sciatica symptoms in almost all the cases, as well as of the lumbar pain, and there was a $60 \%$ improvement in the claudication cases. With regard to complications, we would like to point out two cases due to the technique, one because of the wrong positioning of the screws and the other due to the rupture of the pedicle. There were two cases of subcutaneous seroma and two late subclinical infections.

Conclusions. The dynamic neutralisation obtained using this system, should not be considered as an arthrodesis. Treatment using Dynesys enlarges the population of patiens candidates for surgery to who initially do not apparently need a standard fixation, but who raise doubt regarding the application of techniques without instrumental support, incorporating the functionality concept as opposed to restricting movement. This system can be defined as a disc prosthesis fitted 

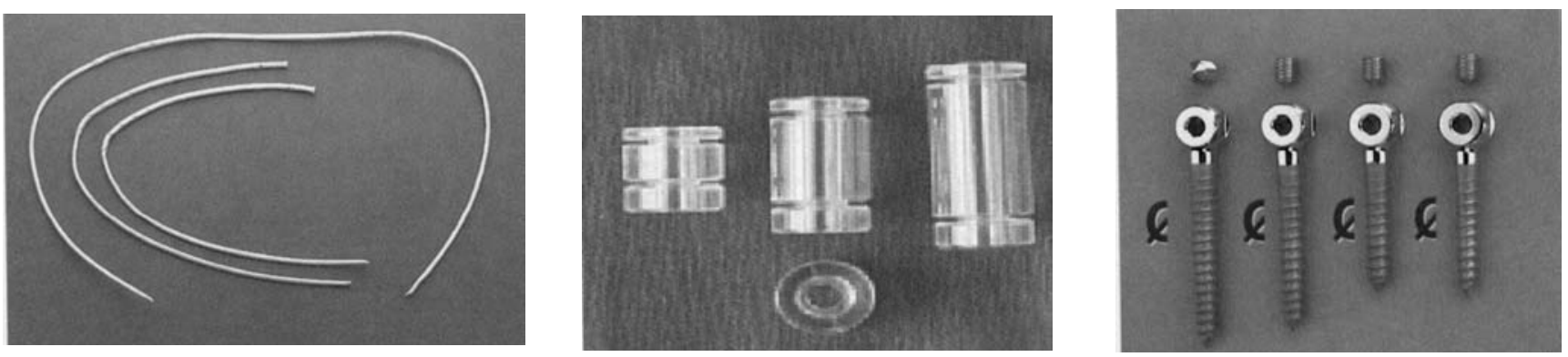

Figuras 1,2 y 3.

externally to the disc. We have obtained good results in the majority of our patients, although we believethut the follow-up should be increased.

KEY WORDS: Lumbar instability. Degenerative lumbosacraI pathology. Dynamic stabilisation. Dynesys neutralisation system.

\section{Introduccion}

La historia del tratamiento de la patología degenerativa de la columna lumbar nos ha hecho recorrer un gran número de técnicas más o menos invasivas con dos objetivos fundamentales como son, por un lado la descompresión de las estructuras neurológicas y, por otro la de conseguir una estabilidad de los segmentos vertebrales afectos, laminectomías, discectomías, técnicas percutáneas por un lado, fijaciones lumbares con y $\sin$ apoyo instrumental, corpectomías etc. todas encaminadas a buscar el control mecánico de la columna reduciendo lo síntomas doloro$\operatorname{sos}^{7-9,13,14}$.

Hemos tenido la oportunidad de utilizar un sistema de nuevo concepto, que trata de conseguir esos principios de estabilidad y reducción del cuadro doloroso, pero con la conservación de la funcionalidad de los elementos dinámicos que se integran en el raquis lumbo-sacro ${ }^{21}$.

Este sistema denominado Dynesys, consta de un elemento espaciador cilíndrico (Sulene pcu) que asegura la estabilidad primaria del sistema absorbiendo las fuerzas de carga, un cordón estabilizador (Sulene pet) que absorbe las fuerzas de tensión, ya que por el trenzado del mismo no permite la extensión, pero sí la flexión y tornillos pediculares de anclaje del sistema de diferentes tamaños realizados con Protasul-100 que permiten la fijación del cordón y favorecen la compresión de los espaciadores modulares. (Figs. 1, 2 y 3$)$.

Los conceptos de funcionalidad contra los de restricción en el movimiento nos hicieron pensar que podía ser un nuevo enfoque en el tratamiento de la patología degenerativa lumbar y, tras intervenir 94 casos aplicando este sistema, presentamos los resultados de los mismos, donde hemos observado mejoras significativas y definitivas en la situación clínica de estos pacientes ${ }^{22}$.

\section{Enfoque anatomo-patologico}

En la evolución natural de la enfermedad discal degenerativa, la lumbalgia es el primer síntoma de la presencia de una discopatía. Esta discopatía se desarrolla progresivamente y puede conducir a distintos estadíos de prolapso de disco que, después de una fase de desestabilización mecánica del trípode funcional (disco y facetas), puede causar el colapso de la vértebra superior dentro de la inferior ${ }^{1}$. Después de este hundimiento de la vértebra superior, la discopatía puede conducir, bien a una fusión espontánea asintomática, o bien a una estenosis (lateral o central) que, en combinación con la espondilolistesis artrítica, es muy dolorosa para el paciente.

En esta evolución de la desestabilización, hay una fase durante la cual las ocasionales lesiones y deformidades son reducibles e incluso reversibles. La lesión mecánica inicial del disco y su posterior evolución pueden visualizarse con ayuda de las imágenes de la RMN en los grados Modic. Con la RMN pueden reconocerse el edema inflamatorio provocado en primer lugar, la creación de tejido graso y la esclerotización final ${ }^{15,16,18}$.

La degeneración discal tiene un impacto sobre las facetas, que va desde pérdida del espacio articular, con deformación de las mismas hasta la osteofitosis hipertrófica, que será el origen de la estenosis lateral y central.

Además del disco, los ligamentos posterior y capsular y las carillas articulares han resultado ser las principales fuentes nociceptoras conducentes al dolor. Por tanto el objetivo del sistema Dynesys es la realineación y re-estabilización de los segmentos próximos a la anatomía funcional ${ }^{5,17}$.

En la evolución de esta fase dinámica, creemos que una re-estabilización puede llevar al segmento intervertebral a un estado más anatómico, en el cual puede tener lugar una curación y, en consecuencia, alcanzar un estado antiálgico. El sistema Dynesys posibilita una estabilización real con un rango controlado de movimiento mediante:

1. Una neutralización dinámica que absorbe las cargas no fisiológicas en compresión y flexión-extensión y que 


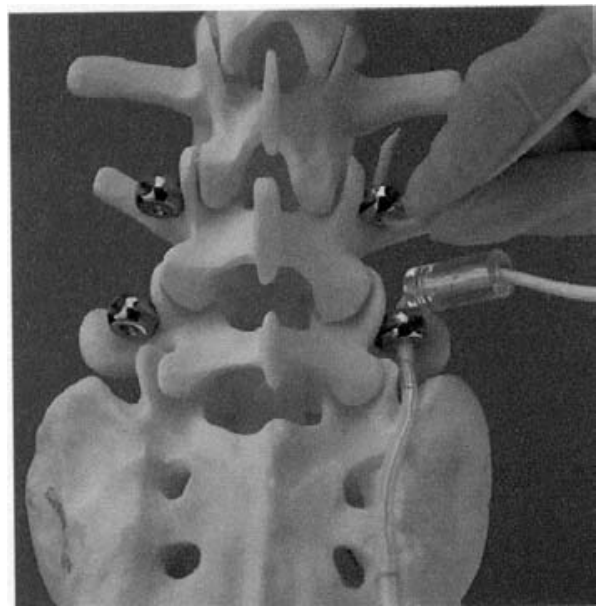

Figuras 4 y 5 .

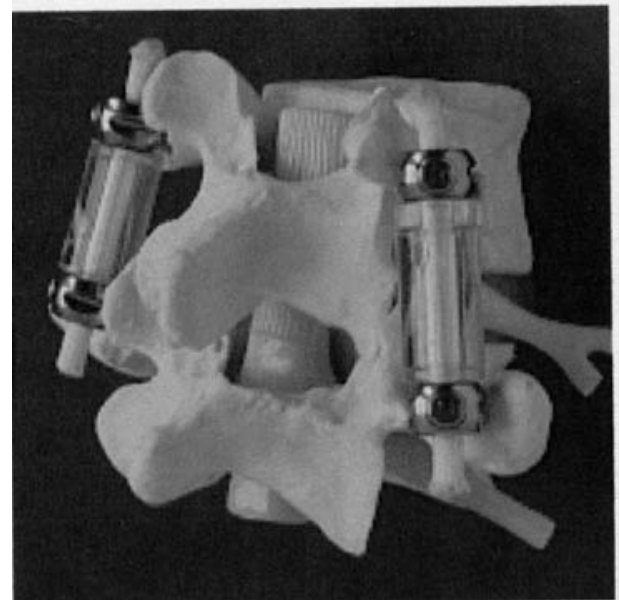

Figuras 6 y 7.

suprime los movimientos parasitarios.

2. Los segmentos espinales pueden ser recolocados en una ubicación anatómica más fisiológica que elimina el dolor $^{19}$.

\section{Material y metodos}

La muestra se compone de 94 pacientes intervenidos desde Marzo de 2002 hasta Diciembre de 2003. La edad se reparte entre 26 y 68 años con una cifra media de 46.4 años, de los que corresponden 62 al sexo masculino y 32 al femenino.

Se ha realizado un test de Oswestry pre y postquirúrgico con cifras que van del $56.8 \%$ al $21.4 \%$ respectivamente. Los estudios radiológicos que se han analizado son RMN y radiología simple pre y postoperatorias, comparando perfiles discales y su repercusión sobre las raices correspondientes, los signos directos e indirectos de estabilidad lumbar pre y postoperatoria, la colocación de los tornillos pediculares y la medida de la distancia entre ellos, en
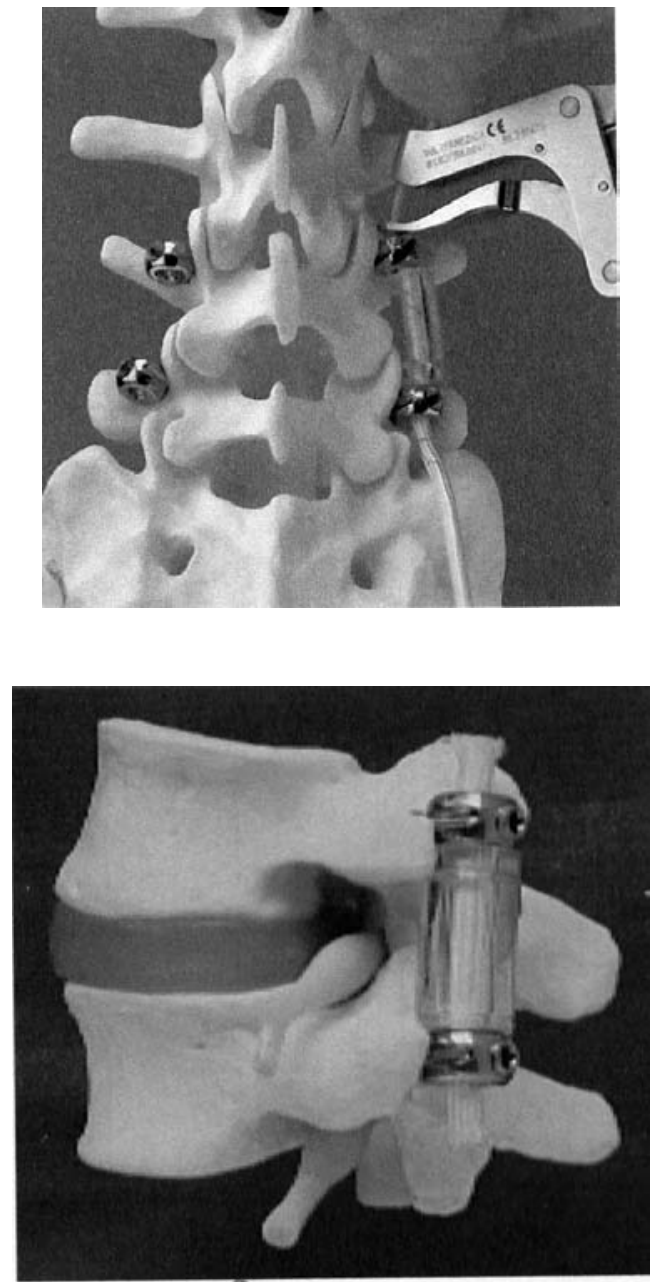

sucesivos controles. También se han valorado posibles cambios en los signos Modic y en la rehidratación del disco intervertebral.

En cuanto a la técnica quirúrgica, hemos utilizado el abordaje estándar por línea media lumbar hasta identificar la cápsula articular procurando no lesionarla. En los casos que debemos practicar una discectomía se procedió de forma habitual hasta liberar los elementos neurales. A continuación se colocaron los tornillos en el ángulo transverso-facetario, se tunelizó el cordón estabilizador siempre comenzando por el nivel inferior, para colocar posteriormente el cilindro espaciador cuya longitud hemos medido anteriormente (Figs. 4 y 5).

En general, y salvo necesidad de distracción específica, hemos distraído una media de 2-3 mm. en ambos lados, comenzando por el lado problema, midiendo el lado contralateral al problema, una vez colocado el sistema en el lado de la lesión, ya que dicha medida puede estar modificada. El cordón estabilizador es fijado por tornillos de presa, que se colocan en la cabeza del tornillo 
transpedicular, mientras se le da al sistema una tensión preestablecida de $300 \mathrm{~N}$ con una pinza de tensión específica. (Figs. 6 y 7).

En los casos en que no se requirió abordaje del canal raquídeo se utilizó el abordaje paravertebral transmuscular, hasta llegar a localizar la apófisis tranversa y desde esa situación implantar el sistema con ayuda de un novedoso instrumental específico que hace más cómoda la técnica, a la vez que es menos invasiva, con sus consiguientes ventajas de ahorro de tiempo, reducción de herida quirúrgica $\mathrm{y}$, sobre todo, de disminución del dolor postoperatorio y conservación funcional de la musculatura.

\section{Datos clínicos}

La patología intervenida se ha determinado tomando como base la clasificación de la patología degenerativa que estableció G. Dubois (esquema I), basándose en la llamada cascada degenerativa de Kikarldy-Willis de 1978. G. Dubois, inspirado en los trabajos de Husson, inició el implante de este sistema en las fases 3 a 6 de aquella clasificación, y que se corresponde con la degeneración discal, estenosis de canal e inestabilidad ${ }^{10}$. La patología más frecuente intervenida ha sido la discopatía degenerativa. (Tabla 1)

\begin{tabular}{|c|c|}
\hline & $\begin{array}{c}\text { Esquema I } \\
\text { FASES DE DESESTABILIZACION }\end{array}$ \\
\hline - I. & Espondiloartrosis + discopatía primaria \\
\hline • II. & Prolapso discal + I \\
\hline • III & Discopatía hipomóvil + I \\
\hline • IV & Inestabilidad funcional +III \\
\hline$\cdot \mathrm{V}$ & Estenosis monosegmentaria + IV \\
\hline$\cdot \mathrm{VI}$ & Estenosis multisegmentaria + IV \\
\hline - VII. & Deformidades estructurales \\
\hline
\end{tabular}

Tabla I

Patología intervenida

$\begin{array}{ll}\text { Hernia discal primaria } & 27 \text { casos } \\ \text { Discopatia degenerativa } & 54 \text { casos } \\ \text { Estenosis de canal } & 13 \text { casos }\end{array}$

La clínica ha correspondido siempre a las lesiones problema. Así, la ciática, lumbalgia, alteración funcional, reducción de la capacidad de deambulación, clínica de pseudoclaudicación, han sido las clásicas sintomatologías que han presentado los pacientes, junto a déficits

\begin{tabular}{lc}
\hline & Tabla II \\
Clínica \\
Lumbalgia & 21 casos \\
Lumbo-ciatica & 63 casos \\
Claudicación & 10 casos \\
\hline \multicolumn{3}{c}{ Tabla III } \\
Niveles tratados \\
\hline L4-L5 & 38 casos \\
L5-S1 & 28 casos \\
L3-L4-L5 & 7 casos \\
L4-L5-S1 & 19 casos \\
L3-L5 D ;L4-L5 1 & 1 caso \\
L2-L3 ; L5-S1 & 1 caso \\
\hline
\end{tabular}

neurológicos, sobre todo a nivel sensitivo y, en algún caso, motor por lesión radicular. Esta sintomatología ha sido fundamentalmente crónica. (Sólo se han tratado 9 casos de evolución aguda. Tabla II).

En cuanto a los niveles intervenidos siempre han sido uno o dos niveles, siendo el más frecuente tratado el espacio L4-L5, solo o en relación con sus vecinos. (Tabla III).

El caso L3-L5 der.; L4-L5 izq. se refiere a la rotura del pedículo L4 derecho, necesitando anclar el tornillo en un nivel superior. El caso L2-L3 ; L5-S1 se refiere a que el espacio intermedio no fue tratado, actuando como si se tratara de dos intervenciones diferentes, por problemas distintos, en el mismo acto quirúrgico, donde existía una hernia discal L5-S1 y un colapso discal con listesis grado 1 y compresión del saco raquideo en L2-L3. Se pensó en esta técnica como alternativa a haber colocado sistema Dynesys en el espacio inferior y una fijación rígida transpedicular en el superior, dado que intraoperatoriamente se observó una recuperable función articular.

\section{Resultados}

En los 94 casos tratados con este método, hemos observado globalmente una mejoría notable de los síntomas que presentaban los pacientes. La valoración que podemos hacer, desde nuestra experiencia, es la desaparición de los síntomas ciáticos en un $96{ }^{\prime} 8 \%$ de los casos, la ausencia de dolor lumbar en un porcentaje del $70 \%$ y una reducción de dichos síntomas suficiente para permitir una vida activa habitual en la mayoría de ellos, habiendo pasado el test de Oswestry preoperatorio de un $56{ }^{\prime} 8 \%$ a un $21^{\prime} 4 \%$ valorado postoperatoriamente. (Tabla IV) 


\section{Tabla IV \\ Resultados}

$\begin{array}{lll}\text { Lumbalgia } & 21 \text { casos: } & 15 \text { asintomáticos, } 5 \text { mejor, } 1 \text { igual } \\ \text { Ciatica } & 63 \text { casos: } & 52 \text { asintomáticos, } 9 \text { mejor, } 1 \text { igual, } 1 \text { peor } \\ \text { Claudicación } & 10 \text { casos: } & 6 \text { mejor, } 3 \text { igual, } 1 \text { peor }\end{array}$

La recuperación para la actividad laboral se ha producido en 76 casos, es decir un $82 \%$. Por actividades podemos ver, lógicamente, que a medida que la actividad laboral es más exigente la reincorporación está más comprometida. (Tabla V). Entendemos por categoría I, actividades como construcción, conducción pesada, carga y descarga, deportistas de élite etc. En la actividad media, actividades tipo doméstico, asistencia, etc., que requiere desplazamiento pero no carga y en el grupo sedentario, labor funcionarial, despacho, etc

Desde el punto de vista de los tiempos empleados, hemos observado una reducción significativa, si comparamos esta técnica con una artrodesis instrumentada estándar, aunque sin poder compararse con las técnicas microquirúrgicas. También se han visto reducidas las pérdidas hemáticas, así como el tiempo de encamamiento, hospitalización y recuperación postquirúrgica, según nuestra propia experiencia. (Tabla VI).

Los resultados radiológicos han podido mostrar una realineación articular así como una realineación vertebral en claudicaciones laterales mediante pequeñas distracciones así como pequeñas reducciones herniarias discales que han hecho que se haya podido conservar la estructura discal en protusiones no extrusas. (Figuras 8 y 9).)

En los controles RMN postoperatorios, a partir de 8-9 meses de seguimiento, se han observado cambios de los

\section{Tabla V}

Categoría I sedentario 20 casos: reincorporacion 19

Categoría II actividad media 30 casos: reincorporación 27

Cateroria III esfuerzos $\quad 44$ casos: reincorporación 30.

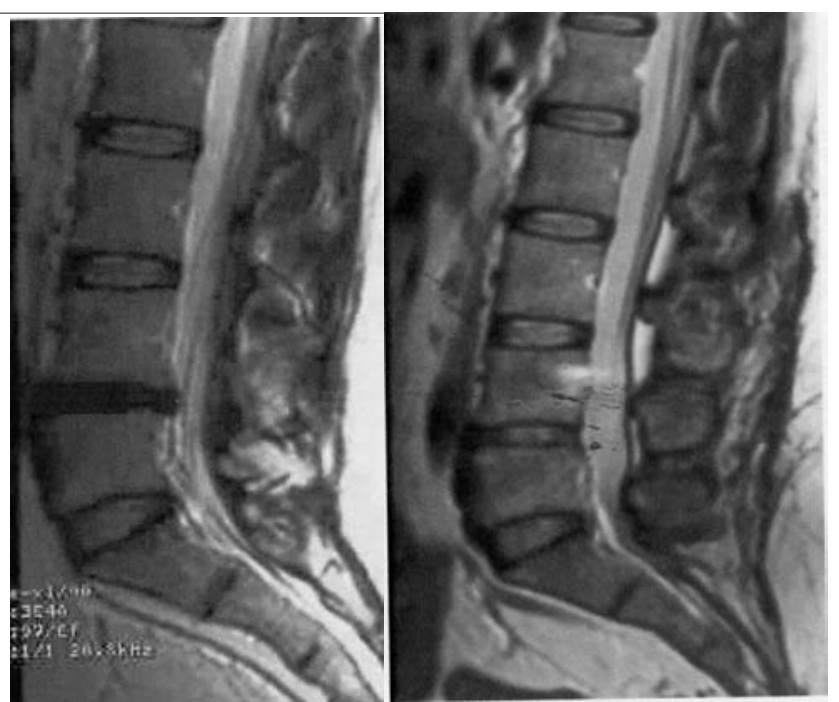

Figuras 8 y 9.

signos de edema en platillos vertebrales (Modic I ) en 6 casos y en 9 de los 20 casos de mayor seguimiento se ha observado signos de rehidratación discal, siguiendo los parámetros del estudio de Specchia. (Figs. 10, 11, 12 y 13).

En los casos en que predomina el compromiso radicular por la extrusión discal se realizó la discectomía en primer lugar y posterior implantación del sistema Dynesys, aunque en ocasiones en que el acceso al espacio fue difícil por el colapso vertebral o por la posición quirúrgica, se optó por realizar primero el implante que, al ampliar el espacio, facilita el abordaje discal. (Figs 14 y 15).

\section{Complicaciones}

No han existido gran número de complicaciones, pero

Tabla VI

Tiempos medios

$\begin{array}{lcll} & \text { Artrodesis } & \text { Dynesys } & \text { Microdiscectomía } \\ \text { Quirófano } & 4 \text { horas } & 2 ' 5 \text { horas } & 1 \text { hora } \\ \text { Hospitalización } & 5 \text { días } & 2-3 \text { días } & 1 \text { día } \\ \text { Recup.laboral } & 6-8 \text { meses } & 1^{\prime} 5-2 ' 5 \text { meses } & 1.5 \text { meses }\end{array}$



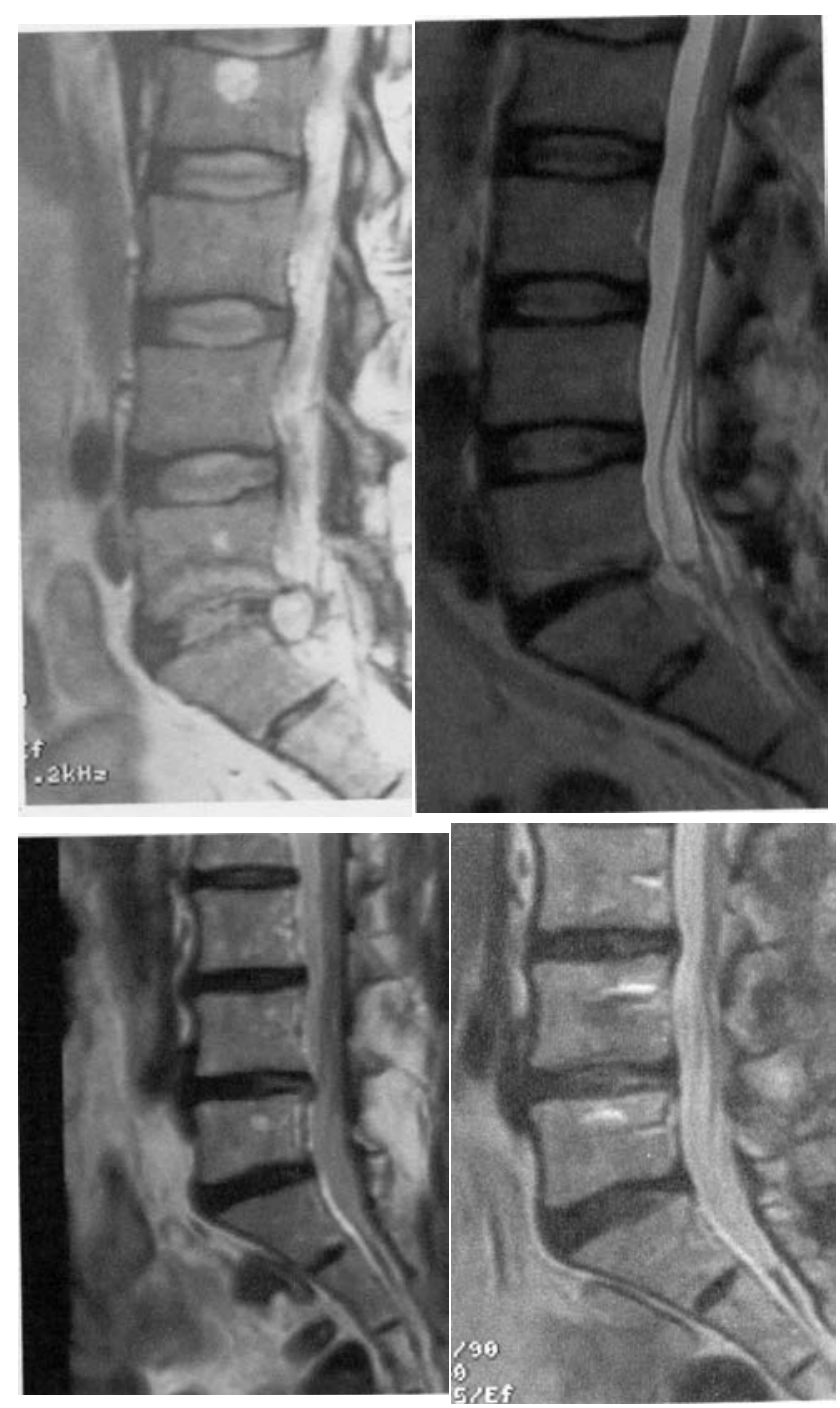

Figuras $10,11,12$ y 13. .

podemos diferenciar las debidas a la técnica y las ajenas a ella. Las complicaciones debidas a la técnica fueron, en un caso, malposición de los tornillos y una fractura pedicular que ya se ha comentado anteriormente. Y las ajenas a ella, dos casos de seroma subcutáneo y dos infecciones subclínicas que obligaron a la retirada del material de instrumentación.

En un alto porcentaje de casos los pacientes han referido unas molestias postoperatorias en territorio femorocutáneo uni o bilateral, que cedieron espontaneamente en dos tres días y que atribuímos al sistema de colocación del paciente en la mesa quirúrgica. Este problema disminuye mediante el almohadillaje a nivel de la espina iliaca anterior.

Con relativa frecuencia, hemos observado la aparición de algias localizadas en region sacroiliaca posiblemente motivadas por el despegamiento muscular a ese nivel, y que en ocasiones necesitaron infiltraciones locales. En
2005; 16: 499-506
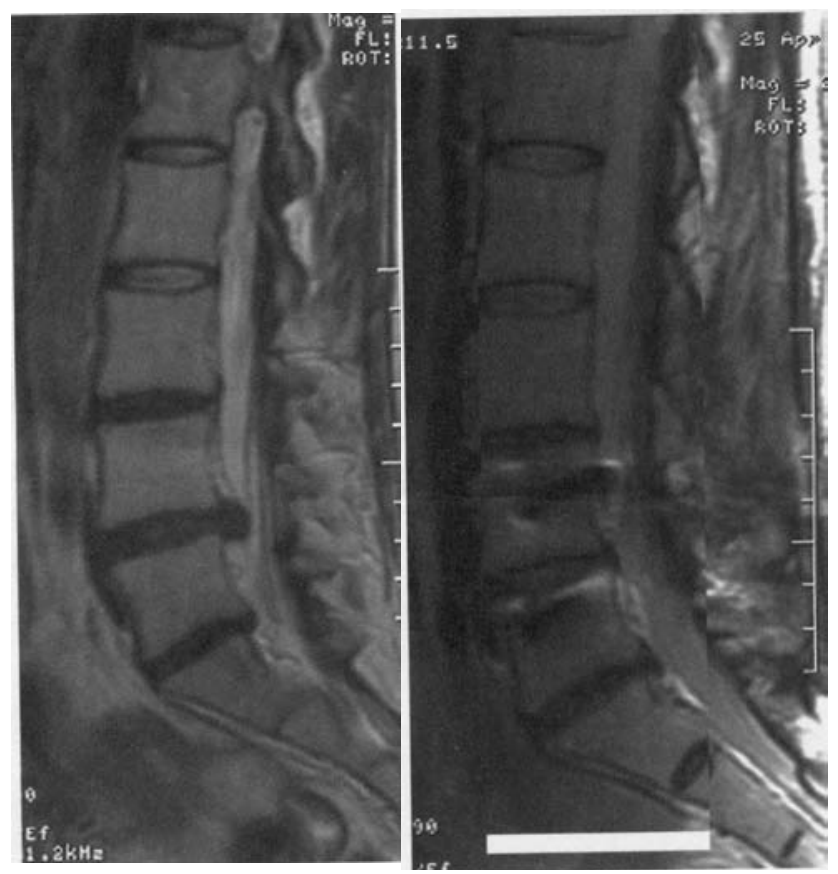

Figuras 14 y $15 .$.

prácticamente todos los casos fue reversible el cuadro salvo en uno de ellos en el que no se observó causa etiológica alguna. Es cierto que una vez adoptado el sistema minimamente invasivo del abordaje paravertebral, este cuadro prácticamente no se presentó lo que nos hace insistir en la técnica de despegamiento muscular como causa de este problema.

\section{Discusión}

En el año 1995, fue implantado por primera vez un sistema de neutralización dinámica para el tratamiento de una patología degenerativa discal. Dubois y cols. ${ }^{2}$ denominaron a este sistema Dynesys (dynamic estabilitation system). En 1998 publicaron sus primeros resultados sobre 50 casos y en el año 2000 su actualización sobre 150 casos. En estos trabajos, los autores, además de presentar la técnica, muestran sus indicaciones basándose en la clasificación que ellos mismos establecen de la patología degenerativa lumbar según el esquema de las enfermedades degenerativas de Kirkaldi-Willis, excluyendo la patología discal primaria, la discopatía hipomovil y las deformidades estructurales ${ }^{11,12}$.

En Mayo del 2000 Stoll y cols publicaron sus resultados, ampliando sus indicaciones a enfermedades degenerativas únicas del disco intervertebral con inestabilidad segmentaria, habiendo presentado en sus series $50 \mathrm{y}$ 76 casos respectivamente, recogidos de estudios multicen$\operatorname{tricos}^{21}$.

En Julio del 2003, Schnake y cols. Presentaron su experiencia en patología degenerativa con estenosis espinal y 
espondilolistesis con un seguimiento de 2 años ${ }^{20}$.

Nuestra serie representa un numero estadístico equiparable a los resultados de otros autores, habiendo indicado el sistema Dynesys en pacientes con patología degenerativa discal, aunque hemos incorporado como indicaciones la protusión discal degenerativa monosegmentaria, donde hemos implantado el sistema sin abordaje del disco, tratando de realinear las facetas. En la patología monodiscal no podemos establecerse un criterio comparativo, ya que en ningún caso previo se había artrodesado, aunque los resultados con Dynesys han sido buenos. Por otra parte, la decisión de utilizar el sistema de neutralización dinámica en deformidades estructurales monosegmentarias creemos que es de gran utilidad en pacientes más jóvenes, puesto que conservan una adecuada funcionalidad de las estructuras articulares.

La ventaja fundamental comparativa con la fijación instrumentada radica precisamente en el concepto fundamental de conservación de los elementos móviles contra los conceptos artrodesantes.

En suma, nuestros resultados son superponibles a los de otras estadísticas que han utilizado esta técnica y consideramos mejores que los tratamientos artrodesantes para la misma patología, incrementando las indicaciones en patología discal degenerativa. Estos resultados los hemos calificado de excelentes en base a criterios de seguimiento clínico (test de Oswestry), cuya valoración nos lleva a un cambio de resultados globales del $56.8 \%$ al $21.4 \%$ definitivo $^{3,4,6}$. Radiologicamente, hemos observado en largos seguimientos signos de rehidratación discal y de atenuación de los hallazgos Modic.

\section{Conclusiones}

1. El sistema Dynesys es un nuevo concepto para el tratamiento de la patología degenerativa discal lumbar, que se basa en el mantenimiento del funcionamiento articular, en contra de la restricción de movimiento de la artrodesis clásica.

2. Técnicamente es de gran facilidad en su colocación, ahorra un tiempo importante en el quirófano, y mejora los tiempos medios de encamamiento y hospitalización.

3. La RM es la exploración de elección para el diagnostico y seguimiento de los pacientes tratados con sistema Dynesys, apoyándose en los estudios de radiología dinámica.

4. Se ha determinado un aumento de las indicaciones en la utilización del sistema. Por un lado en deformidades estructurales monosegmentarias y por otro en patología discal simple no extrusa, donde no se aborda el disco.

5. La técnica permite un segundo tiempo quirúrgico en caso de fracaso, (artrodesis), aunque en nuestra serie no hubo necesidad de ello.

\section{Bibliografía}

1. Ariga, K., Miyamoto, S., Nakase, T., Okuda, S., Meng, W., Yonenobu, K., Yoshikawa, H.: The relationship between apoptosis of endplate chondrcytes and aging and degeneration of the intervertebral disc. Spine; 26: 2414-2420.

2. Dubois, G., de Germay, B., Schaerer, N.S., Fennema, P.: Dynamic Neutralization: A new concept for restabilization. In: Szpalski M, Gunzburg R, Pope MH (Eds), Lumbar Segmental Inestability. Lippincott, Williams and Wilkins, Philadelphia, PA, 1999.

3. Fairbank, J.C.T., Cooper, J., Davis, J.B., O'Brien, J.P.: Oswestry. Low Back Pain Disability Questionnaire. Phisiotherapy, 1980; 66: 271-273.

4. Fairbank, J.C.T., Pynsent, P.B.: The Oswestry Disability Index. Spine, 25: 2940-2953.

5. Freudiger, S., Dubois, G., Lorrain, M.: Dynamic Neutralisation of the lumbar spine confirmed on a new lumbar spine simulator in vitro. Arc. Orthop Trauma Surg, 1999; 119: 127132

6. Fritz, J.M., Irrgang, J.J.: A comparison of a modified Oswestry Low Back Pain Disability Questionnaire and the Quebec Back Pain Disability Scale. Physical Therapy. 2001; 81: 776-788.

7. Graf, H.: Lumbar instability. Surgical treatment without Fusion. Rachis; 412: 123-137.

8. Grevitt, M.P., Gradner, A.D., Spilsbury, J., Shackleford, I.M., Baskerville, R., Pursell, L.M., Hassaan, A., Mulholland, R.C.: The Graf stabilisation system: early results in 50 patients. Eur Spine J; 4: 169-175; discussion p.135.

9. Hadlow, S.V., Fagan, A.B., Hillier, T.M., Fraser, RD.: The Graf ligamentoplasty procedure-comparison with posterolateral fusion in the management of low back pain. Spine; 23: 1172-1179.

10. Husson, J.L., Poncer, R., Polard, J.L.: Dérangement intervertebral acquis (D.I.V.A.). In: Husson JL, Le Huec JC (eds), Restabilisation intersomatique du rachis lombaire. Sauramps Medical, Montpellier, 1996; 13-21.

11. Kirkaldy-Willis, S.H.: Managing Low Back Pain, $3^{\text {rd }}$ edn. Churchill Livingstone, New York, NY, 1992; pp 49-74.

12. Kirkaldy-Willis, W.H., Farfan, H.F.: Instability of the lumbar spine. Clin Orthop; 165: 110-123.

13. Markwalder, T.M., Dubach, R., Braun, M.: Soft system stabilization of the lumbar spine as an alternative surgical modality to lumbar arthrodesis in facet syndrome. Preliminary results. Acta Neurochir; 134: 1-4.

14. Mochida, J., Toh, E., Suzuki, K., Chiba, M., Arima, T.: An innovative meted using the Leeds-Keio artificial ligament in the unstable spine. Orthopedics; 20: 17-23.

15. Modic, M., Pavlicack, W., Weinstein, M., et al.: Magnetic resonance imaging of intervertebral disk disease. Radiology, 1984; 152: 103-111.

16. Modic, M., Ross, J.: Magnetic resonance in the evalua- 
tion of low back pain. Orthop Cin N Am, 1999; 22: 283-301.

17. Mulholland, R.C., Sengupta, D.K.: Rationale, principles and experimental evaluation of the concept of soft stabilization. Eur Spine J, 2002; 11 (Supp1.2): S198-S205.

18. Ross, J., Modic, M., Masaryk, T.: Tears of the annulus fibrosus: assessment with Gd- DTPA-enhanced MR imaging. AJNR Am J Neuroradiol 1989; 10: 1251-1254.

19. Schárer, N., Dubois, G., Braunsweiler, R.: Static and Dynamic Biomechanical Test of a Dynamic Neutralization System for the Spine. En White AA, Panjabi MM (eds), Clinical Biomechanics of the Spine. $2^{\text {nd }}$. Ed. Philadelphia: J.B. Lippincott, 1990: 19-24.

20. Schnake, K.J., Schaeren, S., Jeanneret, B.: Decompression and Dynamic Stabilization in Spinal Stenosis with Degenerative Spondylolisthesis - 2 years follow-up. IMAST. Roma Julio 2003.

21. Stoll, T.M., Dubois, G., Schwrzenbach, O.: The dyna- mic neutralization system for the spine: a multicenter study of a novel non-fusion system. Eur Spine J, 2002 Oct; 11 Suppl 2: S170-S 178

22. White, A.A. III, Panjabi, M.M.: Clinical biomechanics of the spine, $2^{\text {nd }}$. Ed. Philadephia: JB Lippincott Co, 1990: 19-24.

Nota: Los autores no tienen ninguna relación económica con la empresa fabricante o suministradora del Dinesys.

Bordes-Monmeneu, M.; Bordes-García, V.; RodrigoBaeza, F.; Sáez, D.: Sistema de neutralización dinámica en la columna lumbar. SISTEMA DYNESYS Experiencia en 94 casos. Neurocirugía 2005; 16:499-506.

Correspondencia postal: M. Bordes Monmeneu, c/ Jorge Juan 24. 46004 Valencia. 